\title{
Doped copper oxygen planes in a clean habitat
}

\author{
Observation of small Fermi pockets protected by clean $\mathrm{CuO}_{2}$ sheets of a \\ high- $\mathbf{T}_{c}$ superconductor \\ Authors: So Kunisada et al \\ Science 369 833-838 (2020).
}

\author{
Recommended with a Commentary by Dung-Hai Lee, University \\ of California, Berkeley
}

The precise route through which the antiferromagnetic Mott insulating phase evolves into the high-temperature superconducting phase still puzzles the copper-oxide high temperature superconductor research community. For example, upon exiting the antiferromagnetic phase, the sign and the magnitude of Hall number $\left(n_{H}\right)$ is consistent with the density of doped holes $p$ (see Fig.1(a) adapted from[2]), not the expected value $1+p$, namely the average number of holes per unit cell. The discrepancy can be caused by the Fermi surface reconstruction due to some kind of broken symmetry. Alternatively, in the absence of any symmetry breaking, this can signify the presence of topological order!

The paper by Kunisada et al sheds considerable light on this important question. The key to their success is the growth of a $T_{c}=65 \mathrm{~K}$ cuprate crystal that has five copper-oxygen planes per unit cell (the chemical formula is $\mathrm{Ba}_{2} \mathrm{Ca}_{4} \mathrm{Cu}_{5} \mathrm{O}_{10}(\mathrm{~F}, \mathrm{O})_{2}$, see Fig.1(b))[1]. This is complemented by a high-resolution laser ARPES study, plus quantum oscillation, transport, and NMR measurements. The five $\mathrm{CuO}_{2}$ planes can be divided into three groups, two outer planes (OP), two middle-inner planes (IP1) and one inner-most planes (IP0). Because the dopant layers are in contact with the two outer planes OP only, it is claimed that both the doping level and the disorder decreases progressively from OP $\rightarrow$ IP1 $\rightarrow$ IP0.

The Fermi surface image is shown in Fig.1(c) where the open Fermi surface segment (Fermi arc) is attributed to the outer planes, and the two closed Fermi surfaces are attributed to the middle-inner planes (larger Fermi pocket) and the inner-most planes (smaller Fermi pocket). Due to inter-layer hopping, one expects the slight splitting of the IP1 and OP bands. However due to the finite resolution such splitting can be hard to detect experimentally. The geometry and the location of IP0 and IP1 are consistent with the Fermi pockets due to antiferromagnetic order. This claim is supported by the NMR measurement of local magnetic moment[3]. By the measuring the Fermi pocket areas associated with IP0 and IP1, the estimated hole density are $\sim 0.04$ and $\sim 0.09$, respectively. This is consistent with their quantum oscillation result. The fact that the observed pockets are hole pockets is consistent with the Hall coefficient measurement.

Both the OP and IP1 shows a superconducting gap but not IP0. Surprisingly the energy gap associated with IP1 is found to be larger than that associated with OP (see Fig.2(a) and 
(a)

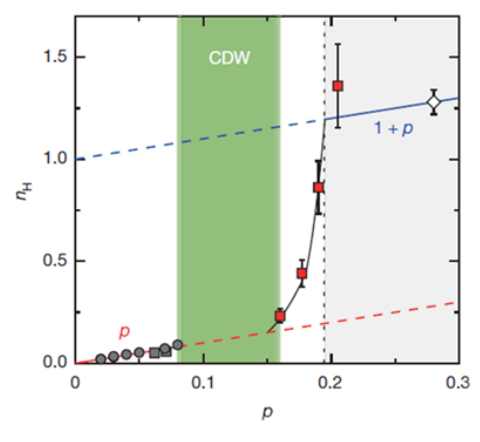

(b)

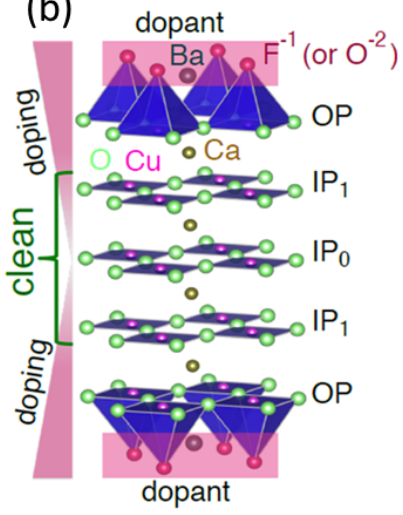

(c)

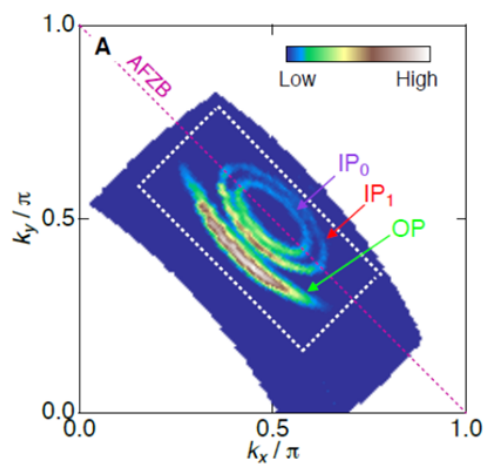

Figure 1: (a) Figure adapted from Ref.[2]. The green shaded region is associated with the appearance of "charge density wave" order where the Hall number is negative, signifying electron dominated transport. The structure of the five-layer compound $\mathrm{Ba}_{2} \mathrm{Ca}_{4} \mathrm{Cu}_{5} \mathrm{O}_{10}(\mathrm{~F}, \mathrm{O})_{2}$. As one goes from the outer to the inner planes, the doping density and disorder both decrease. (c) The Fermi surface imaging near the nodal direction.

Fig.2(b)). This argues against the scenarios that the superconductivity in the middle-inner planes is proximity induced by the outer planes. Thus superconductivity and antiferromagnetism coexists in the middle-inner planes - Cooper pairing simply gaps out the remnant Fermi pockets caused by the antiferromagnetic order. On both IP1 and OP the superconducting gap is consistent with the $d_{x^{2}-y^{2}}$ symmetry (see Fig.2(b)).

Interestingly the energy-distribution curve (EDC) associated with the inner-most plane is sharper than that associated with the middle-inner planes which is in turn sharper than the EDC of the outer planes. The author explains this fact by the decreasing disorder from OP $\rightarrow$ IP1 $\rightarrow$ IP0 (which is supported by the width of NMR lines[3]), and also to the suppression of antiferromagnetic fluctuation due to the long-range order in the inner planes. Significantly, it is found that for IP1 and IP0 the electronic structure near the antinodes $(\pi, 0)$ is very different from that found in the outer planes and also from the that found in other cuprates. More specifically the saddle point is missing, and a parabola disperses down below $-1 \mathrm{eV}$. Therefore, the pseudogap (with the energy scale $\sim 100 \mathrm{meV}$ ) states that known to emerge around $(\pi, 0)$ cannot develop in the inner planes.

To summarize, the results of Kunisada et al suggest, in the clean limit, the lightly doped Mott insulator exhibit coexisting superconducting and antiferromagnetic order. As the doping level increases the gap at the antinodes rapidly decreases to a value $\sim 100 \mathrm{meV}$ and pseudogap appears. The results of Kunisada et al leaves many questions open for future investigations. In the following we list a few examples. First, it remains to be seen whether 

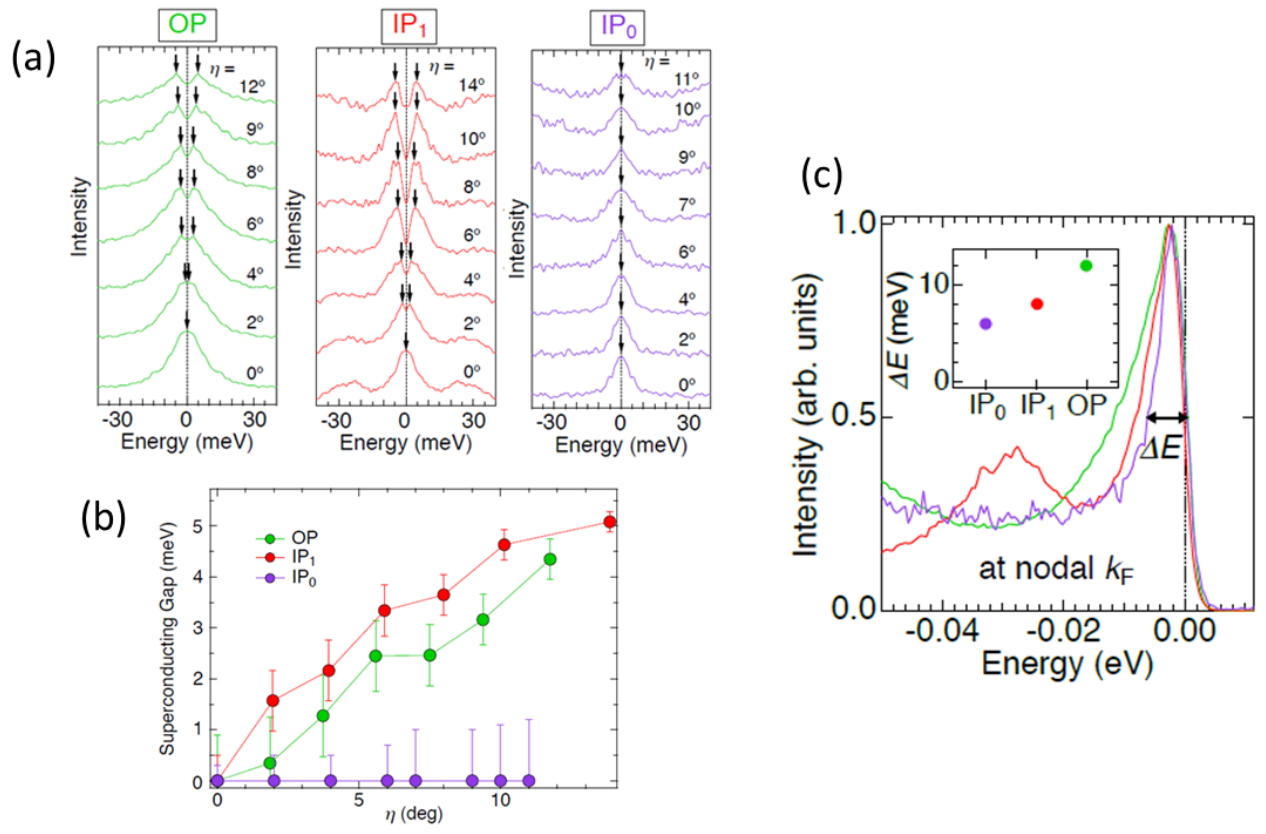

Figure 2: (a) The symmetrized energy distribution curves associated with different angle. Here $\eta$ is the angle between the nodal direction and the direction of the cut. (b) The plot of the superconducting gap as a function of $\eta$. (c) The energy distribution curves associated with OP,IP1 and IP2. Note that the broad peak around $-0.03 \mathrm{eV}$ in the red spectrum comes from the band associated with IP0.

the $n_{H} \approx p$ normal state Hall number observed in all lightly doped superconducting cuprates also signify the coexisting antiferromagnetic order. As far as we know, there is no definitive evidence for such coexistence. One suggestion from the current paper is that this can be due to disorder. Second, the fact that superconductivity is stronger in the IP1 is interesting. On the surface it is at odds with the notion that antiferrmagnetic fluctuation trigger Cooper pairing. Because the antiferromagnetic fluctuation in the OP should be stronger than that in the IP1, where the fluctuation is suppressed by the long-range order. Third, it will be interesting to do the self-energy analysis for the quasiparticle peaks in IP0 and IP1, to see whether they follow the Landau Fermi-liquid behavior. Fourth, it would be interesting to further dope the multi-layer compound to monitor how the Fermi pockets evolves into Fermi arcs. Finally, it remains open what happens at the so-called "pseudogap quantum critical doping" $p \sim 0.2$ in Fig. 1(a)) where the Hall number rapidly climb up to the expected value $1+p$.

\section{References}

[1] Earlier ARPES study of 4 layer $\mathrm{Ba}_{2} \mathrm{Ca}_{3} \mathrm{Cu}_{4} \mathrm{O}_{8} \mathrm{~F}_{2}$ (without the observation of the Fermi pockets) can be found in Yulin Chen et al, Phys. Rev. Lett. 97, 236401 (2006).

[2] S. Badoux et al, Nature 531, 210-214(2016). 
[3] S. Shimizu et al., Phys. Rev. B 85, 024528 (2012). 